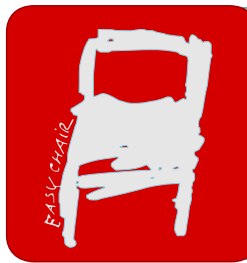

EPiC Series in Health Sciences

Volume 4, 2020, Pages 188-192

CAOS 2020. The 20th Annual Meeting of the International Society for Computer Assisted Orthopaedic Surgery

\title{
Patient-Specific Safe Zone Based on Daily Positions and Range of Motion*
}

\author{
Wistan Marchadour ${ }^{12}$, Guillaume Dardenne ${ }^{12}$, Aziliz Guezou-Philippe ${ }^{13}$, \\ Christian Lefèvre ${ }^{13}$, and Eric Stindel ${ }^{123}$ \\ 1 Laboratory of Medical Image Processing (LaTIM) - INSERM UMR 1101 - SFR IBSAM, Brest, \\ France \\ 2 Regional University Hospital Center of Brest (CHRU), Brest, France \\ 3 University of Western Brittany (UBO), Brest, France
}

\begin{abstract}
Cup orientation is a challenging step in total hip arthroplasty (THA), to ensure comfort of the patient and durability of the prosthesis. The safe zone defined by Lewinnek is commonly used for cup orientation, but it is nowadays being questioned because it is not patient-specific.

We propose to define a new safe zone for cup orientation, that considers patient-specific parameters such as the pelvic tilt and the range of motion (ROM) of the leg.

We developed a software that easily computes a unique safe zone from these parameters in different daily positions, ensuring a cup orientation without risks of prosthetic impingement.
\end{abstract}

\section{Introduction}

The success of total hip arthroplasty (THA) is highly conditioned by the prosthetic cup orientation. To reduce the risks of impingement and dislocation, surgeons follow the recommendation of Lewinnek's safe zone [5], i.e. $40^{\circ} \pm 10^{\circ}$ of inclination and $15^{\circ} \pm 10^{\circ}$ of anteversion, regarding the anterior pelvic plane (APP). However, the safe zone is today controversial [3, 6]: the recommended cup orientations do not take into account patient-specific parameters.

We propose a more patient-specific safe zone, which considers the patient daily positions, as well as the leg range of motion (ROM). We will first detail the method for the computation of this multi parameter safe zone, and then present the software we developed.

\section{Materials and Methods}

\subsection{Patient-specific Safe Zone}

The proposed safe zone considers (1) the daily hip behavior of the patient, obtained from the measurements of the pelvic tilts in supine, standing, and sitting positions, and (2) the desired

*This study received funding from the endowment fund INNOVEO of the University Hospital of Brest.

F. Rodriguez Y Baena and F. Tatti (eds.), CAOS 2020 (EPiC Series in Health Sciences, vol. 4), pp. 188-192 
ROM for six basic movements of the leg (flexion/extension, abduction/adduction, internal/external rotation) in these daily positions.

For each patient daily position (supine, standing, sitting), we compute one prosthetic safe zone from the corresponding desired ROM, regrouping all anatomical cup orientations that will not generate a prosthetic impingement after the operation (Figure 1). The resulting three safe zones are then superposed together. The final safe zone is the common impingement-free zone of the previous ones.

Each position's safe zone is computed by geometrically moving the head and stem in the cup, according to the considered parameters, and by saving the pairs of anatomical cup anteversions and inclinations at the limit of impingement.

\subsection{Required Parameters for Software}

To easily present the proposed safe zone, we developed a software that requires eight inputs, classed in three categories : pelvic tilts, prosthetic parameters and the ROM.

Pelvic Tilts. They give us information on the patient hip motion in three daily positions: supine, standing, and sitting.

Pelvic tilt is defined as the angle between APP and the frontal plane, while APP is the resulting plane of both anterior superior iliac spines (ASIS) and pubic symphysis (PS) points. Literature already showed us that the pelvic tilts can wildly change from one position to another [4].

The pelvic tilts can be acquired without invasive technology or precision loss, using the echography based navigation system developed by Dardenne et al. [1].

Prosthetic Parameters. For simplification of the mathematical system, the prosthesis set is approximated as a perfect ball joint, the cup as an hemisphere, and the stem as a cylinder.

The prosthetic values then come from specifications of the chosen prosthesis model:

- Radius of the prosthetic head;

- Radius of the prosthetic stem;

- Opening angle of the cup;

- Side of the hip to undergo surgery.

We also add parameters of prosthetic pose, corresponding to the stem orientation after placement:

- Stem inclination, defined as the angle between the stem axis and the longitudinal axis of the patient;

- Stem antetorsion, defined as the angle between the frontal axis of the patient and the stem axis projected on the transverse plane. 


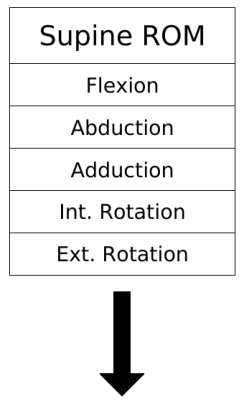

Supine Safe Zone
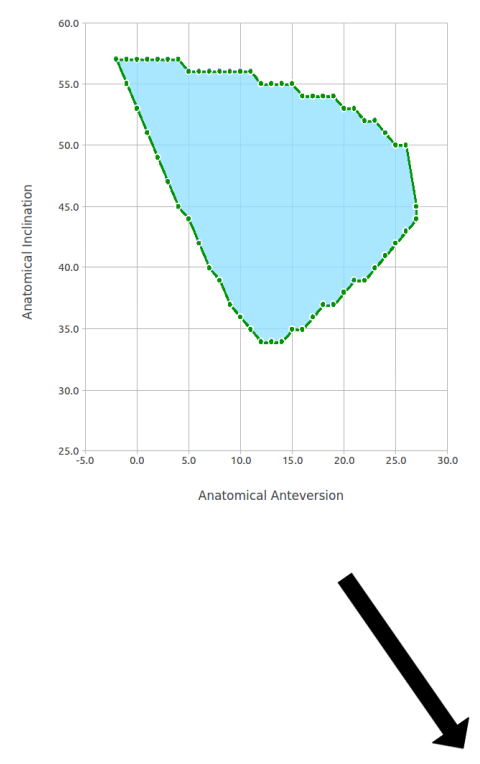

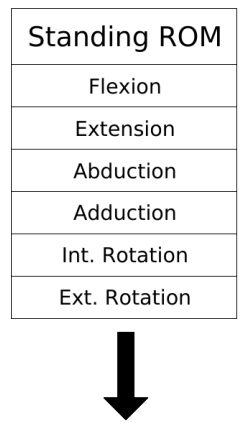

Standing Safe Zone

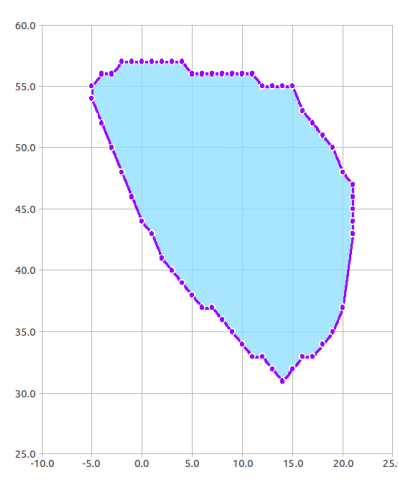

Anatomical Anteversion

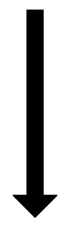

Final Safe Zone

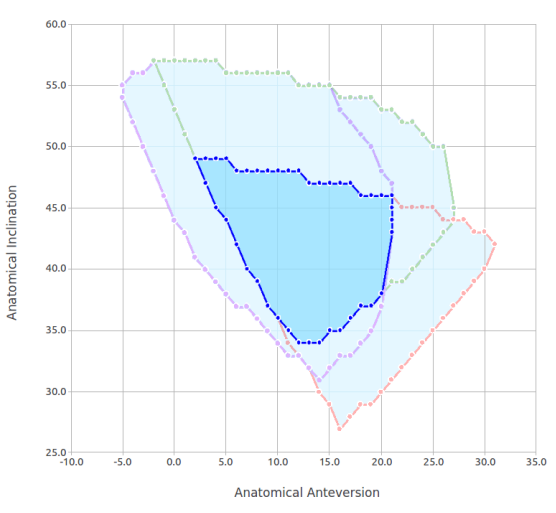

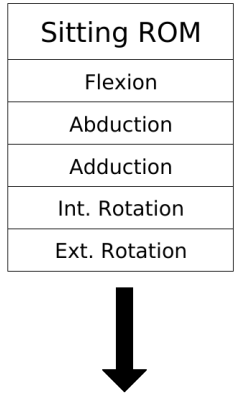

Sitting Safe Zone

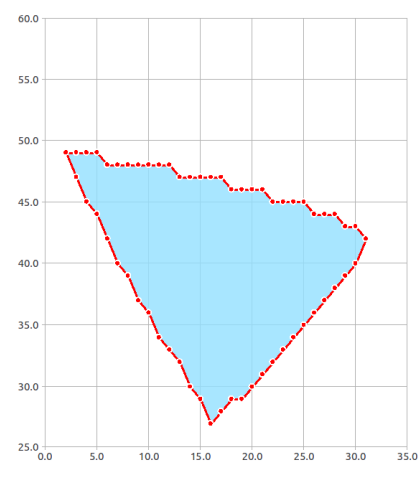

Anatomical Anteversion

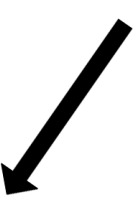


Range of Motion. As the patient must be able to perform daily activities normally, we use the ROM to include the patient ability to make six basic movements: flexion/extension, abduction/adduction, internal/external rotation, in different positions as described above (supine, standing and sitting). Exceptions are made for leg extension in supine and sitting positions, being physically impossible. In supine and standing positions, the internal rotation is realized around the patient frontal axis, after a preliminary leg flexion of $90^{\circ}$.

The complete ROM consists of sixteen movement maximum angles. In the software, the ROM is initialized with default values from the literature [8,9], although the surgeon can always modify it according to the other parameters.

\section{$2.3 \quad$ Tests}

The software has been used to compute the patient-specific safe zone for a group of thirty patients undergoing THA, whose pelvic tilts were previously acquired in the necessary positions.

\section{Results}

The resulting safe zone of one patient is presented as an example in Figure 2.
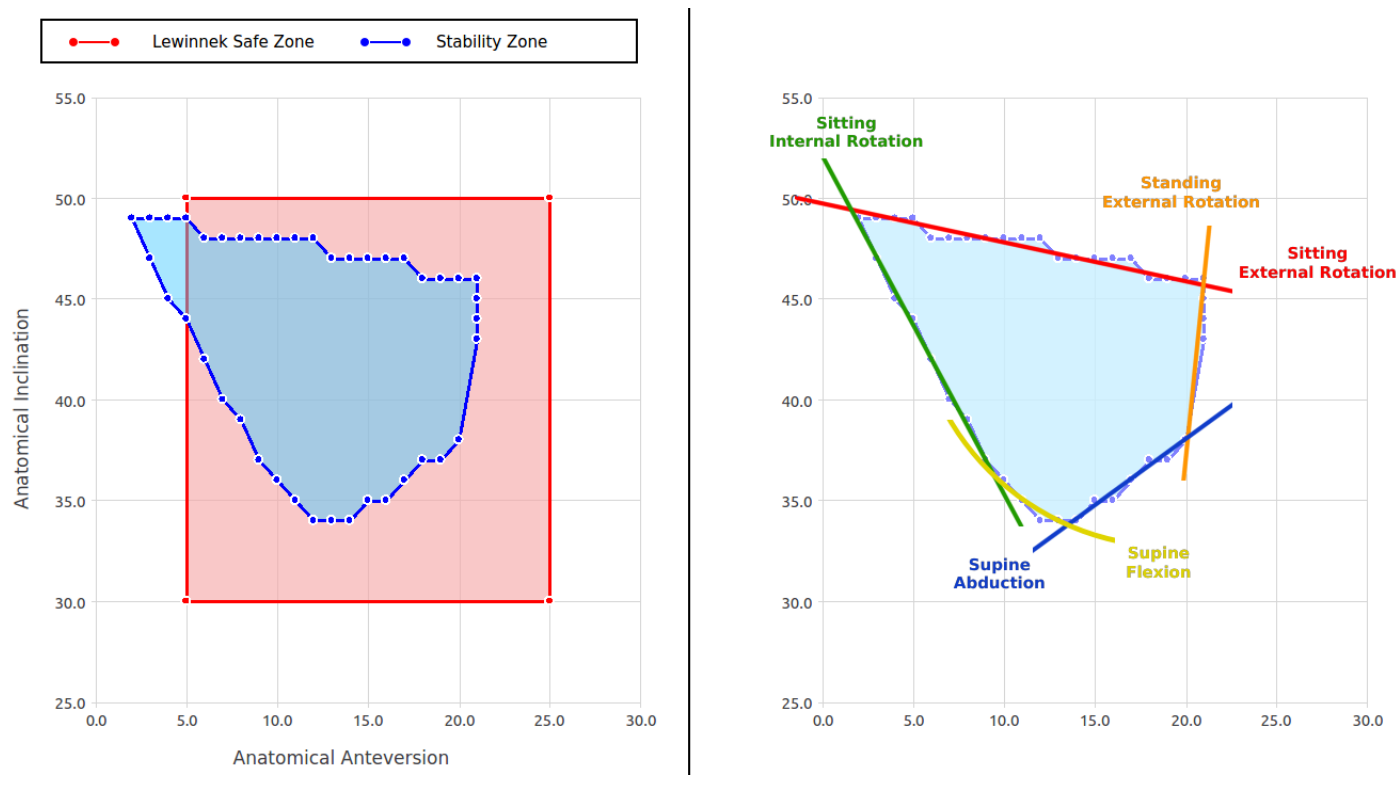

Figure 2: On the left, the resulting graph of the software. The stability zone (blue) is compared to Lewinnek safe zone (red). On the right side, detail on how ROM movements influence the stability zone.

On the left side, the computed new safe zone is compared to Lewinnek safe zone. The new safe zone is irregular, as every parameter influences the result, so it is also unique for the patient. The Lewinnek safe zone is wider, and some recommended cup orientations are excluded from the new zone, involving a very high risk of impingement or dislocation for the prosthesis for some movements. 
On the right side of Figure 2, we detail the influence of the movements on the prosthetic safe zone. Every positions and almost every movement influences the safe zone, highlighting the importance of including the combination of these parameters to our method.

The prosthetic safe zone represents all the anatomical cup orientations (anteversion/inclination) that respect all the parameters values, with the impingement as its limit. Theoretically, if the chosen cup orientation is inside the zone, the patient shall never experience a dislocation due to a prosthetic impingement with the given information.

\section{Discussion}

We developed a software for anatomical cup orientation in THA, that includes pelvic tilts in daily positions. The parameters are acquired with a non-invasive navigation system, and the software generates a unique safe zone for the patient.

Some authors also worked on patient-specific safe zone for cup orientation. Hsu et al. [2] proposed a similar approach, but only keeping the pelvic tilt for supine position. Palit et al. [7] tried to include bony architecture to the list of parameters, using CT scans to determine dislocations risks. The approach is interesting but the hip motion is still not taken into account in the calculations, and the measurements are made with an invasive navigation method.

The patient-specific safe zone software has already been tested with in silico conditions. Our future work will include validation for in vitro environment.

\section{References}

[1] Guillaume Dardenne, Jean-Philippe Pluchon, Hoel Letissier, Aziliz Guezou-Philippe, Romain Gerard, Christian Lefèvre, and Eric Stindel. Accuracy and precision of an ultrasound-based device to measure the pelvic tilt in several positions. Journal of Ultrasound in Medicine, oct 2019.

[2] Juliana Hsu, Matias de la Fuente, and Klaus Radermacher. Calculation of impingement-free combined cup and stem alignments based on the patient-specific pelvic tilt. Journal of Biomechanics, 82:193-203, jan 2019.

[3] Takashi Ishida, Yutaka Inaba, Naomi Kobayashi, Naoyuki Iwamoto, Yohei Yukizawa, Hyonmin Choe, and Tomoyuki Saito. Changes in pelvic tilt following total hip arthroplasty. Journal of Orthopaedic Science, 16(6):682-688, 2011.

[4] Burkhard Lembeck, Otto Mueller, Patrik Reize, and Nikolaus Wuelker. Pelvic tilt makes acetabular cup navigation inaccurate. Acta Orthopaedica, 76(4):517-523, 2005.

[5] G E Lewinnek, J L Lewis, R Tarr, C L Compere, and J R Zimmerman. Dislocations after total hip-replacement arthroplasties. The Journal of Bone 63 Joint Surgery, 60(2):217-220, 1978.

[6] William S. Murphy, Ho Hyun Yun, Brett Hayden, Jens H. Kowal, and Stephen B. Murphy. The Safe Zone Range for Cup Anteversion Is Narrower Than for Inclination in THA. In Clinical Orthopaedics and Related Research, volume 476, pages 325-335. Lippincott Williams and Wilkins, feb 2018.

[7] Arnab Palit, Richard King, Yolanda Gu, James Pierrepont, David Simpson, and Mark A. Williams. Subject-Specific Surgical Planning for Hip Replacement: A Novel 2D Graphical Representation of 3D Hip Motion and Prosthetic Impingement Information. Annals of Biomedical Engineering, 47(7):1642-1656, jul 2019.

[8] Karl H. Widmer and B. Zurfluh. Compliant positioning of total hip components for optimal range of motion. Journal of Orthopaedic Research, 22(4):815-821, 2004.

[9] Fumihiro Yoshimine. The safe-zones for combined cup and neck anteversions that fulfill the essential range of motion and their optimum combination in total hip replacements. Journal of Biomechanics, 39(7):1315-1323, 2006. 